\title{
Planar Negative Refractive Index Media Using Periodically $L-C$ Loaded Transmission Lines
}

\author{
George V. Eleftheriades, Senior Member, IEEE, Ashwin K. Iyer, Student Member, IEEE, and Peter C. Kremer
}

\begin{abstract}
Recent demonstrations of negative refraction utilize three-dimensional collections of discrete periodic scatterers to synthesize artificial dielectrics with simultaneously negative permittivity and permeability. In this paper, we propose an alternate perspective on the design and function of such materials that exploits the well-known $L-C$ distributed network representation of homogeneous dielectrics. In the conventional low-pass topology, the quantities $L$ and $C$ represent a positive equivalent permeability and permittivity, respectively. However, in the dual configuration, in which the positions of $L$ and $C$ are simply interchanged, these equivalent material parameters assume simultaneously negative values. Two-dimensional periodic versions of these dual networks are used to demonstrate negative refraction and focusing; phenomena that are manifestations of the fact that such media support a propagating fundamental back-ward harmonic. We hereby present the characteristics of these artificial transmission-line media and propose a suitable means of implementing them in planar form. We then present circuit and full-wave field simulations illustrating negative refraction and focusing, and the first experimental verification of focusing using such an implementation.
\end{abstract}

Index Terms - Artificial dielectrics, backward waves, focusing, left-handed media (LHM), metamaterials, negative permeability, negative permittivity, negative refractive index, periodic structures.

\section{INTRODUCTION}

$\mathbf{I}_{\mathrm{s}}^{\mathrm{N}}$ THE LATE 1960s, Veselago proposed that materials with simultaneously negative permittivity and permeability are physically permissible and possess a negative index of refraction [1]. Veselago termed these Left-Handed Media (LHM), because the vectors $\mathbf{E}, \mathbf{H}$, and $\mathbf{k}$ would form a left-handed triplet instead of a right-handed triplet, as is the case in conventional, Right-Handed Media (RHM). His conceptual exploration of this phenomenon revealed that, through negative refraction, planar slabs of such media would cause light or electromagnetic radiation to focus in on itself. Although it has been known for some time that arrays of thin metallic wires, by virtue of their collective plasma-like behaviour can produce an effectively negative dielectric permittivity, it was not clear as to

Manuscript received April 4, 2002; revised August 7, 2002. This work was supported by the Natural Sciences and Engineering Research Council of Canada.

The authors are with The Edward S. Rogers Sr. Department of Electrical and Computer Engineering, University of Toronto, Toronto, ON, Canada M5S 3G4 (e-mail gelefth@waves.utoronto.ca; iyer@waves.utoronto.ca; claus@waves.utoronto.ca).

Digital Object Identifier 10.1109/TMTT.2002.805197 how to produce a simultaneously negative permeability. The recent development of the Split-Ring Resonator (SRR) by Pendry et al. [2] was successful in this effort. Subsequently, three-dimensional (3-D) electromagnetic artificial dielectrics (metamaterials), consisting of an array of resonant cells, each comprised of thin wire strips and split ring-resonators, were developed to synthesize the simultaneously negative permittivity and permeability required to produce a negative refractive index, and, indeed, successfully demonstrated reversed refraction [3], [4]. However, the metamaterials presented in [3] and [4] are bulky 3-D constructions, which are difficult to adapt for $\mathrm{RF} /$ microwave device and circuit applications. They consist of loosely coupled unit cells that rely explicitly on the split-ring resonance to synthesize a negative magnetic permeability. Consequently, the structures can achieve a negative index of refraction only within a narrow bandwidth. Furthermore, when applied to wireless devices at RF frequencies the split ring-resonators have to be scaled to larger dimensions, which, in turn would make such devices less compact. It should also be noted that, to date, there has been no experimental verification of the property of focusing inherent to LHM.

This paper offers a fresh perspective on the operation of LHM that enables the design of a new class of metamaterials to synthesize a negative refractive index. The proposed structures go beyond the wire/SRR composites of [2] [4] in that they do not rely on SRRs to synthesize the material parameters, thus leading to dramatically increased operating bandwidths. Moreover, their unit cells are connected through a transmission-line network and they may, therefore, be equipped with lumped elements, which permit them to be compact at frequencies where the SRR cannot be compact. The flexibility gained through the use of either discrete or printed elements enables the proposed planar metamaterials to be scalable from the megahertz to the tens of gigahertz range. In addition, by utilizing varactors instead of capacitors, the effective material properties can be dynamically tuned. Furthermore, the proposed media are planar and inherently support two-dimensional (2-D) wave propagation. Therefore, these new metamaterials are well suited for $\mathrm{RF} /$ microwave device and circuit applications.

The remainder of this paper is divided into four sections. Section II provides an introduction to the theoretical foundations describing lefthanded behaviour in $L-C$ loaded transmission lines, drawing distinctions between the distributed and periodic cases. Section III outlines a method of designing practical periodic $L-C$ loaded transmission lines to synthesize left-handed properties. Section IV presents microwave circuit and full-wave field simulations using these designs. Section V presents the design of planar LHM and the first experimental verification of focusing in LHM. 


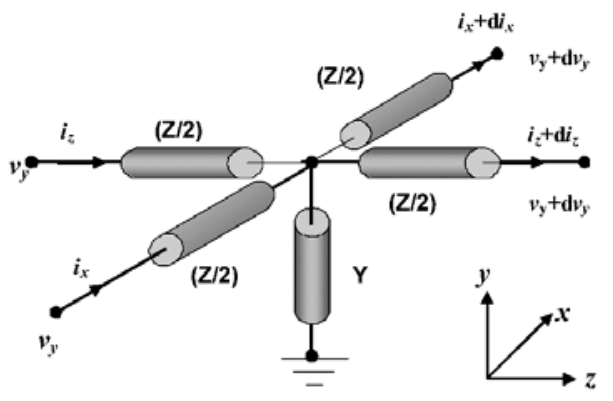

Fig. 1: Unit cell for a 2-D distributed L-C network.

\section{THEORY}

\section{A. Distributed Network Approach}

It is well known that dielectric properties like permittivity and permeability can be modelled using distributed $L-C$ networks. The standard case is the use of periodic versions of these distributed $L-C$ networks to represent free space so that the characteristic impedance of

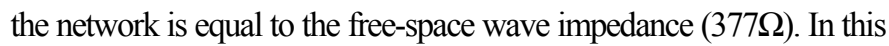
case, the per-unit-length capacitance and inductance can be directly related to the free-space permittivity and permeability, respectively. To illustrate how these material parameters may be effectively derived from a network of generic distributed series impedances and shunt admittances, consider the unit cell pictured in Fig. 1. The 2-D telegrapher's equations representing the distributed structure of Fig. 1 can be expressed as

$$
\frac{\partial v_{y}}{\partial z}=-i_{Z} Z \frac{\partial v_{y}}{\partial x}=-i_{x} Z
$$

and

$$
\frac{\partial i_{z}}{\partial z}+\frac{\partial i_{x}}{\partial x}=-v_{y} Y
$$

Combining (1) and (2) yields

$$
\frac{\partial^{2} v_{y}}{\partial x^{2}}+\frac{\partial^{2} v_{y}}{\partial z^{2}}+\beta^{2} v_{y}=0 \quad \beta= \pm \sqrt{-Z Y}
$$

where $\beta$ is the propagation constant.

It is now appropriate to map field components to the voltages and currents in the medium. In a thin homogeneous isotropic medium, a quasi-static transverse magnetic $\left(\mathrm{TM}_{y}\right)$ solution (i.e., assuming a weak $y$-variation) maps $v_{y}$ to $E_{y}$ (by means of the definition of potential difference) $i_{x}$ to $H_{z}$ (by means of Ampere's law) and $i_{z}$ to $-H_{x}$ and, thus, (1) and (2) correspond to the following field equations:

$$
\frac{\partial E_{y}}{\partial x}=-j \omega \mu_{S} H_{z} \frac{\partial E_{y}}{\partial z}=+j \omega \mu_{S} H_{x}
$$

and

$$
\frac{\partial H_{x}}{\partial z}-\frac{\partial H_{z}}{\partial x}=+j \omega \varepsilon_{s} E_{y}
$$

yielding the effective material parameters as

$$
j \omega \mu_{S}=Z \Rightarrow \mu_{S}=\frac{Z}{j \omega}
$$

and

$$
j \omega \varepsilon_{S}=Y \Rightarrow \varepsilon_{S}=\frac{Y}{j \omega} .
$$

For the case of free space, we require $\mu_{S}=\mu_{0}$ and $\varepsilon_{S}=\varepsilon_{0}$, and we choose $Z=j \omega \mu_{0}$ and $Y=j \omega \varepsilon_{0}$. This implies a medium in a low-pass topology with $L=\mu_{0}(\mathrm{H} / \mathrm{m})$ and $C=\varepsilon_{0}(\mathrm{~F} / \mathrm{m})$, both of which are positive real quantities. The corresponding propagation constant reduces to that of a standard transmission line (in this case, air-filled),

$$
\beta=\sqrt{-Z Y}=\omega \sqrt{L C}=\omega \sqrt{\mu_{0} \varepsilon_{0}}
$$

The resulting phase and group velocities at low frequencies are parallel and given by

$$
v_{\phi}=\frac{\omega}{\beta}=\frac{1}{\sqrt{L C}}=\frac{1}{\sqrt{\mu_{0} \varepsilon_{0}}}=\left(\frac{\partial \beta}{\partial \omega}\right)^{-1}=v_{g} .
$$

That the phase and group velocities are both positive results from the choice of the positive root in (8). The choice is arbitrary since it serves only to select one of two solutions related through a space-reversal or, equivalently, one of the two branches of the corresponding dispersion diagram. That is, in RHM, the phase lags in the direction of the positive group velocity (which, in this case, is parallel to the Poynting vector), a fact that is invariant to the sign selected for the root. In the case of RHM, (9) implies that the refractive index is positive. Indeed, the refractive index can be defined as

$$
n=\frac{c}{v_{\phi}}=\frac{\sqrt{L C}}{\sqrt{\mu_{0} \varepsilon_{0}}}
$$

which is positive and equal to unity for the case of free space. Furthermore, the characteristic impedance of the network is exactly equal to the free space wave impedance, as expected, and as follows:

$$
Z_{0}=\sqrt{\frac{L}{C}}=\eta_{0}=\sqrt{\frac{\mu_{0}}{\varepsilon_{0}}} \cong 377 \Omega .
$$

Naturally, this concept need not be limited to free space; rather, it is applicable to any other homogeneous isotropic dielectric, since we are free to specify the choice of $L$ and $C$. This prompts us to examine the scope of distributed $L-C$ networks for reproducing unique material parameters, including those of LHM. Specifically, Veselago's postulation of a negative permittivity and permeability prompts the question of whether the $L$ and $C$ parameters in a network representation can also be made negative. Naturally, from an impedance perspective, imposing a negative $L$ and $C$ essentially exchanges their inductive and capacitive roles, so that the series inductor becomes a series capacitor, and the shunt capacitor becomes a shunt inductor. The emerging dual structure is easily recognized as having the topology of a high-pass filter network. The resulting equivalent permittivity and permeability can be shown to be negative, using arguments similar to those of the free space case. Consider such a dual high-pass topology, with distributed 
series capacitance $C^{\prime}(\mathrm{F} \cdot \mathrm{m})$ and shunt inductance $L^{\prime}(\mathrm{H} \cdot \mathrm{m})$. Using (6) and (7), we arrive at the following results:

$$
\begin{gathered}
j \omega \mu_{S}=Z=\frac{1}{j \omega C^{\prime}} \Rightarrow \mu_{S}=-\frac{1}{\omega^{2} C^{\prime}} \\
j \omega \varepsilon_{S}=Y=\frac{1}{j \omega L^{\prime}} \Rightarrow \varepsilon_{S}=-\frac{1}{\omega^{2} L^{\prime}},
\end{gathered}
$$

which show that the equivalent material parameters are indeed negative for a dual $L-C$ network. The corresponding propagation constant is related inversely to the frequency,

$$
\beta=-\sqrt{-Z Y}=-\frac{1}{\omega \sqrt{L^{\prime} C^{\prime}}} \cdot
$$

In this case, the phase and group velocities are antiparallel, and are given by

$$
v_{\phi}=\frac{\omega}{\beta}=-\omega^{2} \sqrt{L^{\prime} C^{\prime}}, v_{g}=\left(\frac{\partial \beta}{\partial \omega}\right)^{-1}=+\omega^{2} \sqrt{L^{\prime} C^{\prime}}
$$

where the choice of the negative root in (14) has assured a positive group velocity (in this case also parallel to the Poynting vector). This implies that, in LHM, the phase leads in the direction of the group velocity, or power flow, and the index of refraction should accordingly be negative. Indeed, the corresponding refractive index is negative as follows:

$$
n=\frac{c}{v_{\phi}}=-\frac{1}{\omega^{2} \sqrt{L^{\prime} C^{\prime}} \sqrt{\mu_{0} \varepsilon_{0}}}=\frac{\sqrt{\mu_{S} \varepsilon_{S}}}{\sqrt{\mu_{0} \varepsilon_{0}}} .
$$

This result further justifies the interpretation of (12) and (13) as equivalent effective material parameters. It is also clear that the relationship between the network characteristic impedance and equivalent wave impedance is preserved according to the following equation:

$$
Z_{0}=\sqrt{\frac{L^{\prime}}{C^{\prime}}}=\eta=\sqrt{\frac{\mu_{S}}{\varepsilon_{S}}}
$$

It should be noted that, although the parameters themselves are negative, their spectral derivatives remain positive due to the negative, inverse-square dependence on frequency, so that the total stored time-averaged energy [5], expressed by the following:

$$
W=\frac{\partial\left(\mu_{S} \omega\right)}{\partial \omega}|\mathbf{E}|^{2}+\frac{\partial\left(\varepsilon_{S} \omega\right)}{\partial \omega}|\mathbf{H}|^{2}
$$

remains positive, and causality is not violated.

The results indicated by (15) are familiar because they seem to describe the well-known phenomenon of the backward wave, in which the Poynting and wave vectors are oppositely directed [6]. Indeed, Veselago has alluded to the presence of backward waves in his definition of left handedness [1]. Thus, the above development seems to suggest that there exists a multitude of materials capable of demonstrating negative refraction phenomena solely as a consequence of the fact that they support backward waves.

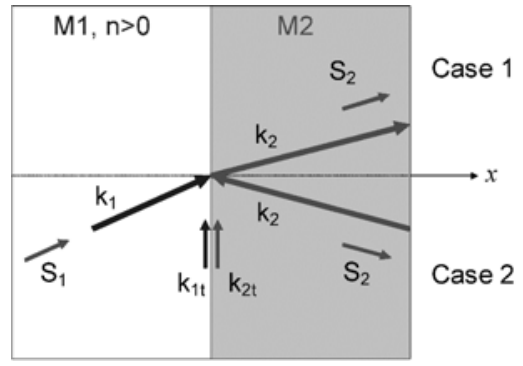

Fig. 2: Phase matching at the interface between a right-handed medium M1 and a generic medium M2.

We shall now use these backward waves to assert that the proposed dual $L-C$ networks do demonstrate negative refraction. Consider a two-medium interface, where medium 1 (M1) exhibits a positive index of refraction and medium 2 (M2) is regarded as generic for the moment. The complete arrangement is depicted in Fig. 2. A plane wave originating in M1 and impinging on the interface with wave vector $\mathbf{k}_{1}$ establishes a refracted wave in M2 with wave vector $\mathbf{k}_{\mathbf{2}}$ such that the tangential wave-vector components $\mathbf{k}_{\mathbf{1 t}}$ and $\mathbf{k}_{\mathbf{2 t}}$ are equal. This phase matching is a generalized form of Snell's Law, and that it specifies constraints only on the tangential components of the wave vectors presents some interesting consequences. There is, in fact, the freedom of two possibilities for the normal component of $\mathbf{k}_{\mathbf{2}}$ : the first and usual case, in which $\mathbf{k}_{\mathbf{2}}$ is directed away from the interface, and the second case, usually reserved for reflected waves, in which $\mathbf{k}_{\mathbf{2}}$ is directed towards the interface. These two cases are represented as Case 1 and Case 2 in Fig. 2. We may further invoke the constraints imposed by the conservation of energy, so that normal components of the Poynting vectors $\mathbf{S}_{\mathbf{1}}$ and $\mathbf{S}_{\mathbf{2}}$ remain in the positive $x$-direction through both media.

It is clear that Case 1 depicts the familiar situation in which M2 is a conventional positive-index medium (depicted as denser than M1 in Fig. 2). However, if M2 is a medium supporting propagating backward waves (LHM), then it is, indeed, true that the wavevector $\mathbf{k}_{\mathbf{2}}$ must be directed opposite to the Poynting Vector $\mathbf{S}_{2}$, so that power is propagated along the direction of phase advance. Therefore, refraction in media that support backward waves must be described by the second case, for which it may be argued that power is refracted through an effectively negative angle, which, indeed, implies a negative index of refraction.

According to [1] and [7], electromagnetic waves from a point source located inside a RHM can be focused by a planar LHM slab, as depicted in Fig. 3. Geometrical optics indicates that, for a planar interface, the focal point is generally dependent on the incident angle. For the case of negative-index optics, some elementary manipulations of Snell's Law at the first interface depicted in Fig. 3 show that the distance of the internal focal point from the interface is given by the following:

$$
f_{1}\left(\theta_{\text {inc }}, \theta_{\text {refr }}\right)=s_{1} \cdot\left|\frac{n_{L H M}}{n_{R H M}}\right| \cdot \frac{\cos \left(\theta_{\text {refr }}\right)}{\cos \left(\theta_{\text {inc }}\right)}
$$

where $s_{1}$ is the distance of the source from the interface, as depicted in Fig. 3, and $\theta_{i n c}$ and $\theta_{r e f r}$ are the absolute values of the angles of incidence and refraction, respectively. From the 


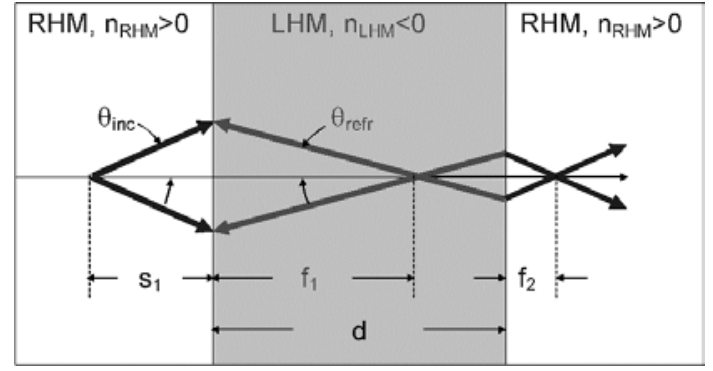

Fig. 3: Internal and external focusing using a LHM slab of thickness d.

second interface, the distance of the external focal point can be given by equation (20),

$$
f_{2}\left(\theta_{i n c}, \theta_{r e f r}\right)=s_{1} \cdot\left(\frac{d}{f_{1}\left(\theta_{i n c}, \theta_{r e f r}\right)}-1\right)
$$

where $d$ is the slab thickness. Consequently, both the internal and external focal points generally depend on the angle of incidence, suggesting inherent spherical aberration. However, it is clear that they may be made angle-independent in the special case for which the relative refractive index is -1 . In this case, $\theta_{i n c}$ and $\theta_{\text {refr }}$ become equal, and the internal and external focal points become unique. This observation seems to corroborate the findings of [8], which state that, unless the relative refractive index is -1 , only a paraxial focus can be defined.

\section{B. L-C Loaded Transmission Line Network Approach}

The previous discussion has confirmed that distributed $L-C$ networks possessing a high-pass topology support backward waves; a property that enables these media to demonstrate negative refraction and focusing phenomena, and therefore, permits us to refer to them as left handed (in Veselago's terminology). However, any practical implementation of these structures at RF/microwave frequencies must be a periodic one, and must therefore possess a non-vanishing cell dimensionality. Herein, we consider a practical planar design that periodically loads a host transmission line network with discrete reactive elements. The finite-length transmission lines provide the needed dimensionality, but at the same time perturb the homogeneity of the distributed system to the extent that the previously described treatment requires modification.

A rigorous understanding of the behaviour of the proposed LHM structures can be gained through their dispersion characteristics. Only the one-dimensional (1-D) case is treated here for simplicity, but the results can be easily extended to two dimensions. The desired dispersion relation can be obtained through standard periodic analysis based upon the 1-D, symmetric unit cell of Fig. 4. Here, $Z$ and $Y$ represent discrete loading elements since the cell dimension $d$ is explicitly provided for by the transmission line segments $\left(Z_{0}, k\right)$.

In the previous discussion on distributed $L-C$ networks, the frequency response permitted propagation at all frequencies. However, when these finite-dimension cells are periodically cascaded, the corresponding dispersion diagram develops a band structure. The propagation constant $\beta$ can be determined

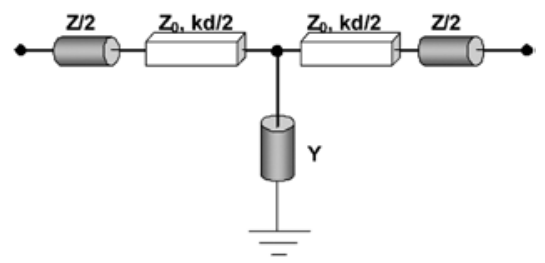

Fig. 4: Unit cell for the one-dimensional periodic L-C network featuring the host transmission line medium $\left(\mathrm{Z}_{0}, \mathrm{k}\right)$.

using the standard procedure for 1-D periodic analysis of microwave networks [9], [10]. The resulting dispersion relation is

$$
\cos \beta d=\cos \theta+\frac{Z Y}{2} \cos ^{2} \frac{\theta}{2}+\frac{j}{2}\left(\frac{Z}{Z_{0}}+\frac{Y}{Y_{0}}\right) \sin \theta
$$

where we have used $\theta=k d$. It is clear from (21) that when the period of the structure is infinitesimally small, i.e., $\cos \theta \rightarrow 1$ and $\sin \theta \rightarrow 0$, the effects of the transmission lines disappear and the medium becomes continuous. This corresponds to the case of a high-pass filter, when operating well within the filter passband. Retaining only the first two terms in the Taylor expansion of the resulting expression, we may determine a first-order approximation for the phase shift per unit cell according to

$$
1-\frac{(\beta d)^{2}}{2} \cong 1+\frac{Z Y}{2} \Rightarrow \beta d=-\sqrt{-Z Y}
$$

yielding the previously obtained result for the distributed $L-C$ network as given by (14). This result describes the phase shift incurred by propagation through the periodic structure over a distance $d$. Making the high-pass substitutions

$$
Z=\frac{1}{j \omega C_{0}}, Y=\frac{1}{j \omega L_{0}}
$$

with $L_{0}$ and $C_{0}$ being the discrete loading elements, (22) gives

$$
\beta d=-\sqrt{-Z Y}=-\frac{1}{\omega \sqrt{L_{0} C_{0}}} .
$$

The reader will recall that, while (14) regards $L^{\prime}$ and $C^{\prime}$ to be distributed parameters (with units henry- $m$ and farad $-m$, respectively), the equivalence between (14) and (24) is established if the cell dimensionality $d$ in (24) is absorbed into $L_{0}$ and $C_{0}$. It should also be noted that, once again, the negative square root is taken in (24) to ensure a positive group velocity as given by (15). The corresponding effective index of refraction is given by

$$
n=\frac{\beta d}{k_{0} d}=\frac{-\sqrt{-Z Y}}{\omega \sqrt{\mu_{0} \varepsilon_{0}} d}=-\frac{1}{\omega^{2} \sqrt{L_{0} C_{0}} \sqrt{\mu_{0} \varepsilon_{0}} d}
$$

where, again, the equivalence between (16) and (25) is established through the cell dimension $d$.

The above development has assumed a unit cell with small dimensions, so that the loading elements can still be treated as distributed parameters. However, in general, the effect of the discrete, periodic loading of the host transmission line medium cannot be neglected. In the full dispersion relation given by (21), 


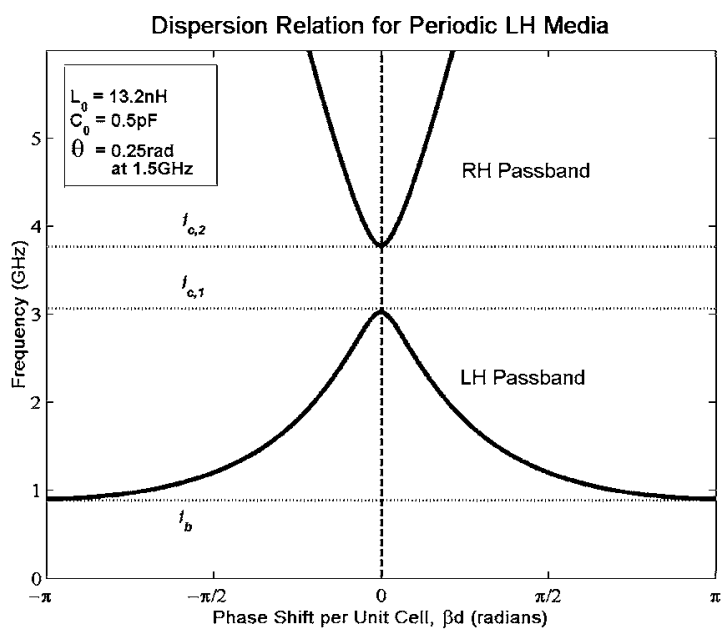

Fig. 5: Dispersion relation for periodically $L-C$ loaded LHM using $L_{0}=13.2 \mathrm{nH}$, $C_{0}=0.5 \mathrm{pF}$, and $\theta=0.25$ radians at $1.5 \mathrm{GHz}$, indicating Bragg frequency $f_{b}$ and stopband limits $f_{C, 1}$ and $f_{C, 2}$.

it is evident that $\beta$ can become periodically complex, forbidding propagation at these frequencies. Choosing $L_{0}=13.2 \mathrm{nH}, C_{0}=0.5 \mathrm{pF}$, and $\theta=0.25$ radians at $1.5 \mathrm{GHz}$, the full dispersion relation of (21) is depicted in Fig. 5. As shown, passbands are observed, separated by a finite stopband. The lowest cut-off (Bragg) frequency associated with this dispersion curve (indicated in Fig. 5 by a dashed line) is approximately determined from the condition $\beta d=\pi$ to be

$$
f_{b}=\frac{1}{4 \pi \sqrt{L_{0} C_{0}}} .
$$

Employing a higher level of precision in the approximation of (21) than (22) permits the analytic determination of the stopband limits

$$
\cos \beta d \cong 1-\frac{\theta^{2}}{2}+\frac{Z Y}{2}+\frac{j}{2}\left(\frac{Z}{Z_{0}}+\frac{Y}{Y_{0}}\right) \theta .
$$

Making the substitutions of (23), with $\theta=k d=\omega d / v$ (where $v$ is the phase velocity in the host medium), and setting $\beta=0$ in (27), the solution of the resulting quadratic equation in $\omega$ yields the desired cut-off frequencies (see Fig. 5) as follows:

$$
f_{c, 1}=\frac{1}{2 \pi} \sqrt{\frac{v}{C_{0} Z_{0} d}}, f_{c, 2}=\frac{1}{2 \pi} \sqrt{\frac{v}{L_{0} Y_{0} d}}
$$

Note that both cutoff frequencies in (28) tend to infinity as the cell dimensionality $d$ approaches zero, thus arbitrarily increasing the bandwidth of the first LH passband (see Fig. 5). Furthermore, closing this stopband by equating these two cutoff frequencies yields the matching condition

$$
Z_{0}=\sqrt{\frac{L_{0}}{C_{0}}}
$$

which suggests that the width of the stopband may be controlled by adjusting the mismatch between the characteristic impedances of the host medium and the loading. It is clear from the lowest passband of this dispersion diagram (the left-hand-side branch in Fig. 5 ) that the phase and group velocities are oppositely directed, implying that this periodic version of the previous distributed structure also supports backward waves. Furthermore, as was explained earlier, the bandwidth of this region can be controlled and substantially widened by selecting a sufficiently small periodicity $d$. The reader will also recognize from Fig. 5 that successive passbands alternately exhibit left or right handedness (using Veselago's terminology) since the concavity of the dispersion surface changes with frequency. Although all periodic structures support an infinite number of forward- and backward-wave spatial harmonics, [6], [9], the dispersion diagram of Fig. 5 shows that the periodic dual $L-C$ network supports a backward-wave fundamental spatial harmonic (extending over the region $-\pi<\beta d<0$ ).

\section{DESIGN}

In [11], a procedure was described for the design of high-pass $L-C$ networks to demonstrate negative refraction. This procedure will be presented in detail herein.

The demonstration of negative refraction requires the realization of an interface similar to that depicted in Fig. 2, so it is necessary to design both LHM and RHM using periodic $L-C$ arrays. The final design therefore consists of two large periodic arrays of 2-D $L-C$ unit cells, each as shown in Fig. 1. One array is constructed using unit cells configured in a low-pass topology to simulate free-space conditions, and the second array is designed according to the proposed dual $L-C$ topology. To prevent reflections at the interface, the characteristic impedances of both media are designed to be equal.

As a starting point to the design, the dimensionless periodic $L-C$ unit cell described by (22) may be employed. However, it is clear from (24) and (25) that, since the reactive elements included in the unit cells are discrete elements, the propagation constant and, therefore, the refractive index, is dependent on the periodicity $d$. Consequently, it is not possible to specify an index of refraction unless the manner of distribution of the discrete elements over $d$ is specified. However, this constraint may be alleviated by specifying a relative index of refraction between the two media, or equivalently the ratio of the phase incurred in each medium through the period $d, \beta_{\mathrm{LHM}} d / \beta_{\mathrm{RHM}} d$. The arrays may therefore be designed by means of circuit simulations using discrete elements, since all dependence on dimension is removed in the ratio.

Choosing a relative refractive index using the ratio $\beta_{\mathrm{LHM}} d / \beta_{\mathrm{RHM}} d$ and a suitable operating frequency, the LHM and RHM capacitors and inductors are specified according to equations (24) and (17), and will hereinafter be referred to as $\left(C_{\mathrm{LHM}}, L_{\mathrm{LHM}}\right)$ and $\left(C_{\mathrm{RHM}}, L_{\mathrm{RHM}}\right)$, respectively. The corresponding cut-off frequencies are then determined and verified to enclose the chosen operating frequency.

Once this starting point is established, it is necessary to account for the effect of the finite-length transmission lines comprising the host medium. The lengths must be chosen to be small enough that the distributed parameters of the lines do not supersede the effects of the loading elements. The process of compensation for the presence of lines will now be described in detail.

The presence of the discrete, periodic loading renders the results of (24) and (25) ineffective in predicting the behaviour of the $L-C$ loaded transmission line medium. It is, therefore, neces- 


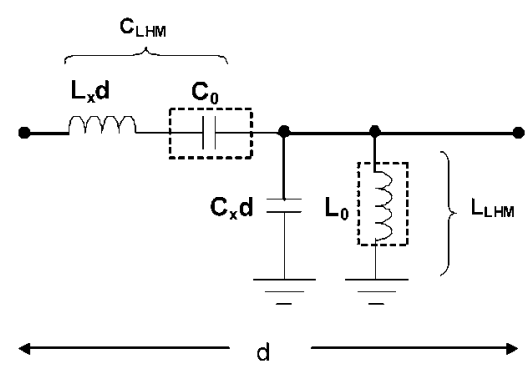

Fig. 6: 1-D unit cell with host transmission line medium embedded as an equivalent series inductor and shunt capacitor.

sary to develop a procedure to modify the values of the discrete loading elements such that the value of $\beta$ is restored, particularly at the frequency of operation, to that obtained in the dimensionless case. That is, the key design idea is to explicitly account for the contribution of the transmission-line parameters in the total inductance and capacitance per unit cell. Specifically, the per-unitlength transmission line parameters of the line $L_{x}$ and $C_{x}$ can be incorporated into the unit cell by modifying the cell as depicted for the 1-D case in Fig. 6.

Here, $C_{0}$ and $L_{0}$ are the new loading elements, and the total impedance in the series and shunt branches give the designed equivalent series capacitance $C_{\mathrm{LHM}}$ and shunt inductance $L_{\mathrm{LHM}}$, respectively, at the design frequency.

The required new series loading capacitance $C_{0}$ can be determined using the following equation,

$$
j \omega L_{x} d+\frac{1}{j \omega C_{0}}=\frac{1}{j \omega C_{L H M}},
$$

which simplifies, through some manipulation, to the desired result

$$
C_{0}=\frac{C_{L H M}}{1+\omega^{2} C_{L H M} L_{x} d} .
$$

Before specifying $L_{0}$, it is important to note that, in the 2-D version of the medium, it is necessary to further compensate for the effect of the host medium transverse to the direction of propagation. This is most clearly illustrated for the case of normal incidence, for which each of the two transverse transmission line segments become opencircuited at their centres, and, therefore, contribute capacitively to the unit cell. This is shown in Fig. 7 from the top-view. Consequently, the loading shunt inductance $L_{0}$ must be appropriately enhanced, and Fig. 6 can be modified, as shown in Fig. 8 .

Here, $Y_{O C}=\left(-j Z_{0} \cot (k d / 2)\right)^{-1}$, and $k$ is the propagation constant in the unloaded medium. The required new $L_{0}$ can then be determined by the following equation:

$$
j \omega C_{x} d+\frac{1}{j \omega L_{0}}+\frac{2}{-j Z_{0} \cot (k d / 2)}=\frac{1}{j \omega L_{L H M}}
$$

which can be reduced to the following simpler relationship:

$$
L_{0}=\frac{L_{L H M}}{1+\omega^{2} L_{L H M}\left(2 C_{x}\right) d}
$$

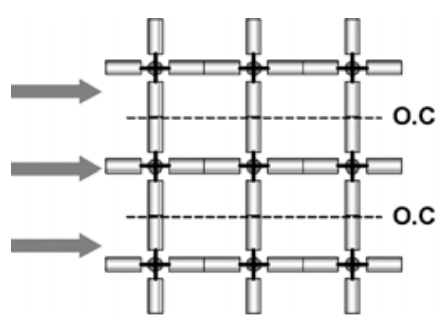

Fig. 7: Top view of transmission line grid, showing open-circuits formed in the medium transverse to the direction of propagation, for the case of normal incidence.

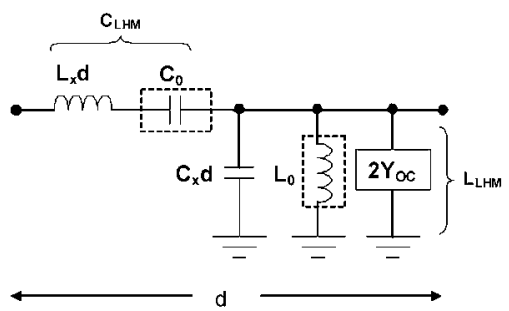

Fig. 8: The one-dimensional unit cell of Fig. 6 with the effect of the transverse host medium represented as a shunt capacitance $\mathrm{Y}_{\mathrm{OC}}$.

through the use of the small angle approximation. That is, for $k d<<2$, the third term on the left-hand side of (32) becomes identical to the first term. It is, therefore, apparent that the capacitive contribution of the two open-circuited stubs in shunt is limited to the effect of their transmission line parameters. Moreover, since $C_{x}$ can be given by $\varepsilon_{r} \mathcal{E}_{0}$, the transverse stubs serve, effectively, to double the relative permittivity of the unloaded medium if they are not compensated for.

The equivalent material parameters of (12) and (13) may now be given by

$$
\begin{aligned}
& \mu_{S}=\mu_{r, L H M} \mu_{0}=-\frac{1}{\omega^{2} C_{L H M} d}=L_{x}-\frac{1}{\omega^{2} C_{0} d} \\
& \varepsilon_{S}=\varepsilon_{r, L H M} \varepsilon_{0}=-\frac{1}{\omega^{2} L_{L H M} d}=2 C_{x}-\frac{1}{\omega^{2} L_{0} d}
\end{aligned}
$$

from which it can be seen that each of these parameters consists of a positive contribution due to the unloaded medium, and a negative contribution due to the loading (corresponding to an effective negative susceptibility).

A similar procedure is followed to compensate for transmission lines in the unloaded medium. In fact the complete spatial dispersion characteristics of these 2-D LHM (and RHM) have been derived but their presentation is beyond the scope of this paper. Instead it suffices to state that, for the examples to follow, the spatial dispersion is negligible and the media can be regarded as homogeneous and isotropic (under TM-wave excitation conditions).

\section{Simulation}

\section{A. Microwave Circuit Simulations}

LHM and RHM arrays were designed as described in the previous section, terminated at their boundaries using resis- 


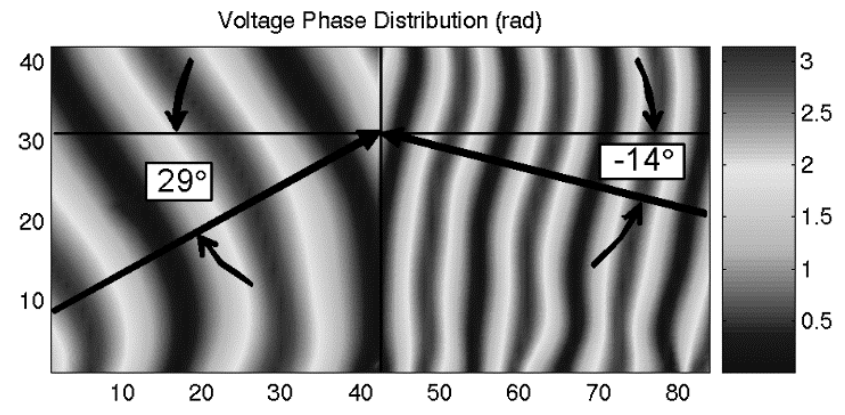

Fig. 9: Plane wave illuminating a RHM/LHM interface at $2 \mathrm{GHz}$ (relative refractive index -2) at an incident angle of $29^{\circ}$. Refraction observed at $-14^{\circ}$. The horizontal axis is labeled according to cell number, and the right-handside vertical scale designates radians.

tors matched to $377 \Omega$, and simulated with a commercial microwave circuit simulator, Agilent-ADS. To verify negative refraction, as illustrated in the phase-matching argument of Fig. 2, a RHM/LHM interface was constructed using $42 \times 42$-cell RHM and LHM arrays with $\beta_{\mathrm{RHM}} d=+0.25$ and $\beta_{\mathrm{LHM}} d=-0.5$, yielding a relative refractive index of -2 . The unit cells comprising the arrays were designed to be 5 -mm square, and consisted of transmission-line segments and the designed loading elements. The corresponding equivalent, absolute index of refraction of the LHM could therefore be determined by (25) to be -2.4 . To maintain the relative refractive index of -2 , the absolute index of refraction of the RHM was made to be +1.2 . The required loading elements were specified to be $\left(C_{0, \mathrm{LHM}}=297.7 \mathrm{fF}\right.$, $\left.L_{0, \mathrm{LHM}}=32.6 \mathrm{nH}\right)$ and $\left(C_{0, \mathrm{RHM}}=-35.7 \mathrm{fF}, L_{0, \mathrm{RHM}}=1.2 \mathrm{nH}\right)$ using $(31)$ and (33), and their right-handed counterparts, respectively. The RHM shunt loading capacitance is negative (inductive) to provide sufficient compensation for the relatively large shunt capacitance of the transverse stubs, so that the overall shunt reactance is capacitive.

The plane wave excitation $\mathbf{k}_{\mathbf{1}}$ shown in Fig. 2 was created using a series of voltage sources placed along the left-hand-side boundary of the array, sequentially phase-shifted to synthesize the desired tangential wave-vector components $\mathbf{k}_{\mathbf{1}}=\mathbf{k}_{\mathbf{2}}$, thus specifying the wave vectors in both media. One such excitation is shown in Fig. 9, for an incident angle of $\theta_{\mathrm{RHM}}=29^{\circ}$. Since the relative refractive index was designed to be -2 , Snell's Law dictates the angle of refraction to be $14^{\circ}$. Indeed, the steepest phase descent in the LHM is observed at $\theta_{\mathrm{LHM}}=14^{\circ}$ from the normal, verifying Snell's Law for the given design parameters. This circuit arrangement has, therefore, unambiguously demonstrated the phenomenon of negative refraction in L-C loaded transmission lines in a dual (high-pass) configuration.

The conditions for focusing were modeled by exciting a single node inside the RHM and observing the magnitude and phase of the voltages to ground at all points in the LHM. It was expected that a focusing effect would manifest itself as a "spot" distribution of voltage at a predictable location in the LHM.

In the interest of completeness, cases of both positive and negative refraction were examined. In the first case, a $42 \times 21$-cell RHM array was interfaced with another $42 \times 63$-cell RHM array with a relative refractive index of +2 . The source was placed 11 cells into the first RHM. Here,
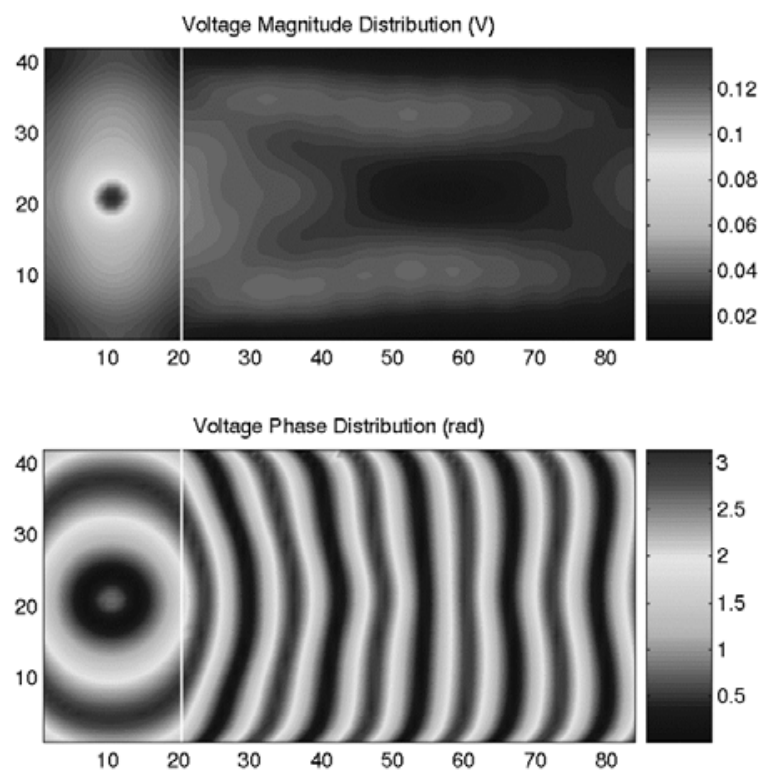

Fig. 10: Point source illuminating a RHM1/RHM2 interface at $2 \mathrm{GHz}$ (relative refractive index +2 , finite air-filled transmission lines with $d=5 \mathrm{~mm}$ included in each unit cell), no focusing observed in either phase or magnitude. The horizontal axis is labeled according to cell number.

focusing was not expected since Snell's Law for positive-index media predicts a continued divergence into the second RHM. Fig. 10 presents magnitude and phase plots of these results, and confirms that the cylindrical wave excitation diverges into the second medium.

The second case consisted of a RHM/LHM interface with a relative refractive index of -2 , using the array dimensions and source location specified above. This arrangement was expected to show focusing inside the LHM. However, the relative refractive index was not designed to be -1 , which implies that the focal point must vary with the angle of incidence. In this case, geometric optics predicts spherical aberration, as illustrated in Fig. 11. As a reference, one can make use of the fact that the paraxial limit of (19) dictates a focus in the LHM at twice the distance of the source from the interface, or near cell 43 of the composite array. Indeed, as shown in Fig. 12, the corresponding magnitude and phase results show focusing in this region, manifested in increased voltage amplitudes (nearly $65 \%$ of the source amplitude). Equally dramatic is the reversal of the concavity of the wave fronts at both the RHM/LHM boundary and the expected focal point. Furthermore, minimal reflections are observed near the upper and lower edges of the array, indicating that the direction of power flow has truly been reversed.

\section{B. Full-Wave Field Simulations}

The planar left-handed medium can be practically implemented using a network of microstrip lines to serve as the host medium. The corresponding full-wave simulations were performed using the Multiradius Bridge Current (MBC) thin-wire moment-method simulator [12]. The MBC simulator represents microstrip lines and surface patches using long, cylindrical wires of thin radii, and permits dimensionally infinitesimal lumped elements to be embedded in the wire mesh. 


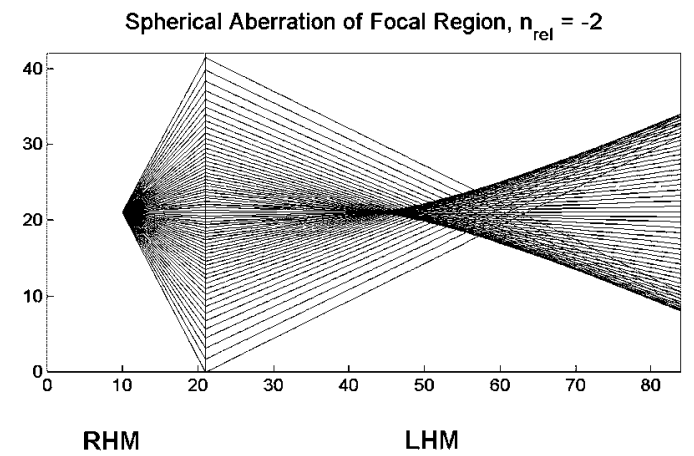

Fig. 11: Spherical aberration of the focal region at an RHM/LHM interface with a relative refractive index -2 .
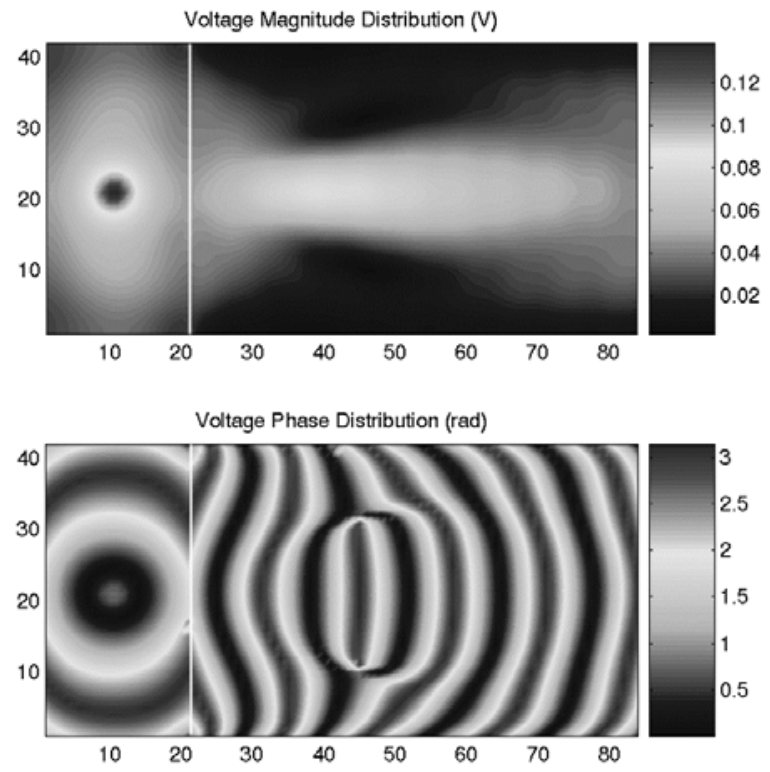

Fig. 12: Point source illuminating a RHM/LHM interface at $2 \mathrm{GHz}$ (relative refractive index -2 , finite air-filled transmission lines with $d=5 \mathrm{~mm}$ included in each unit cell), focusing observed in both phase and magnitude; The horizontal axis is labeled according to cell number.

With forethought to the physical implementation of the LHM structure, the design was specified for a substrate of height $1.524 \mathrm{~mm}$ (60 mil), with a Rexolite dielectric $\left(\mathcal{E}_{r}=2.53\right)$. The equivalent characteristic impedance of the lines comprising the host medium may therefore be approximated by that of a two-wire line, with radius $a_{0}$ and axis-to-axis distance equal to twice the substrate height $d$ (using image theory). This is described by the following relation [6]:

$$
Z_{0}=\frac{\eta_{d}}{\pi} \cosh ^{-1}\left(\frac{d}{a_{0}}\right) \quad \eta_{d}=\frac{\eta_{0}}{\sqrt{\varepsilon_{e f f}}}
$$

where $\eta_{d}$ is indicated to be the wave impedance in the substrate dielectric, $\eta_{0}$ is the free-space wave impedance, and $\varepsilon_{e f f}$ is the effective permittivity of space surrounding the unloaded medium. On this point, it is necessary to note that the MBC simulator is limited in that it makes no provisions for inhomogeneously filled media. Consequently, it was necessary to assume that the proposed LHM structure was embedded in the

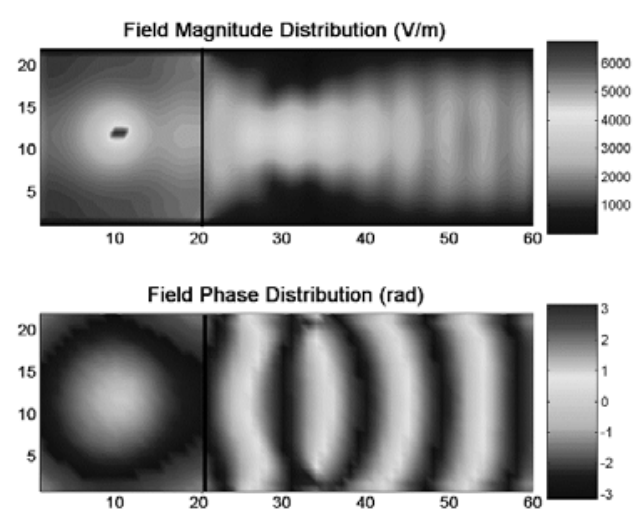

Fig. 13: Vertical electric field distribution due to a voltage source illuminating the interface between an unloaded transmission line grid (RHM with $n=+2.25$ ) and a dual $L-C$ loaded grid (LHM designed for $n=-5.5$ ) with periodicity $d=5 \mathrm{~mm}$ and relative permittivity of space $\mathcal{E}_{r}=2.53$; The horizontal axis is labeled according to cell number.

Rexolite substrate, so that the effective relative permittivity of all space was 2.53. The equivalent radius $a_{0}$ in (36) was chosen to be $0.533 \mathrm{~mm}$ to synthesize a characteristic impedance equal to that of a $400-\mu \mathrm{m}$-wide microstrip line over the same substrate, for which standard quasi-static formulas exist [10]. To be consistent with the arrangement depicted in Fig. 12 for microwave circuit simulation, an RHM is required to supply a cylindrical wave excitation. For this purpose, the MBC simulator used an unloaded wire grid to represent a parallel-plate waveguide. The refractive indices of the parallel-plate medium (nearly frequency independent) and LHM at $1.5 \mathrm{GHz}$ were specified to be +2.25 (corresponding to a grid with period 5-mm square embedded in Rexolite) and -5.5 , respectively, yielding a relative refractive index of about -2.45 . Using (31) and (33), the required loading elements for the 2-D LHM were approximately $L_{0, \mathrm{LHM}}=13.2 \mathrm{nH}$ and $C_{0, \mathrm{LHM}}=0.5 \mathrm{pF}$. These equivalent values suggest an approximate cut-off frequency of $900 \mathrm{MHz}$. Conductor losses were included in the simulations by reducing the nominal bulk copper conductivity by an order of magnitude, i.e., from $57.0 \times 10^{6}$ $\mathrm{S} / \mathrm{m}$ to $5.7 \times 10^{6} \mathrm{~S} / \mathrm{m}$. The final arrangement provided to the simulator consisted of a parallel-plate waveguide, represented by a $20 \times 20$-cell unloaded wire grid, interfaced with a $20 \times 40$-cell $L-C$ loaded LHM grid. A voltage generator was vertically placed at the center of the parallel-plate waveguide, and the approximate relative refractive index of -2.45 suggested that the focal point would become apparent near cell 45 of the composite array. The magnitude and phase of the resulting vertical electric fields are shown in Fig. 13 at $1.5 \mathrm{GHz}$. These results demonstrate that, indeed, focusing occurs in this practical structure as well. The vertical field magnitudes experience a collective peak (once again, nearly $65 \%$ of the source amplitude) and the evolution of the concavity of the wavefronts is consistent with that of the circuit simulations (see Fig. 12). Moreover, by examining the phase incurred in each region per unit distance, the inferred indices of refraction in the RHM and LHM were found to be +2 and -4.44 , respectively, which are close to the designed values. On the other hand, the focal region, determined using the location of inflection of the wavefront concavities, occurs closer to the interface than ex- 
pected, corresponding to a relative refractive index of about -1.4 , instead of the designed relative index of -2.45 . It can be speculated that this discrepancy is related to edge effects as well as the limitations imposed by geometrical optics as applied to the electrically small structure under consideration. Unfortunately, it was not possible to perform simulations on larger grids due to CPU and memory resource limitations. However, the important conclusion to be drawn from the results of Fig. 13 is that this realistic, planar structure unambiguously demonstrates focusing, attesting to its left handedness.

The analysis of Section II was based on the assumption that TM waves would be excited in the structures. This has been verified by the presented full-wave simulations, which showed strong vertical $E$-fields. An examination of the corresponding vertical $H$-fields in the same simulation showed that they were indeed weak and localized compared to the transverse $H$-fields, and there was no indication of systematic phase progression. These results suggest that a TM mode is predominant in the proposed LHM structure.

\section{EXPERIMENT}

\section{A. Experiment Design and Fabrication}

The proposed 2-D $L-C$ loaded transmission line structures are ideally suited to implementation using standard printed circuit board (PCB) fabrication techniques. However, the construction of a proof-of-concept prototype had to rely on manual implementation. Consequently, the prototype designed for the present investigation sought to demonstrate focusing with a minimum number of unit cells. By scaling the results of Fig. 13 (and, further, by verifying through full-wave simulations), it was determined that an LHM array consisting of $11 \times 6$ unit cells (of 5-mm square cell dimension) is sufficiently large to demonstrate a reasonable focal spot.

As in the simulations, the fabricated LHM consisted of $400-\mu \mathrm{m}-$ wide microstrip lines on a 60-mil Rexolite dielectric $\left(\varepsilon_{r}=2.53\right)$ substrate. The designed inductance of $13.2 \mathrm{nH}$ was effectively realized with chip inductors embedded into 1-mm holes drilled into the substrate at the appropriate cell sites. Chip capacitors of $0.5 \mathrm{pF}$ were surface-mounted between gaps etched into the grid lines, and $1-\mathrm{pF}$ capacitors were placed at the array edges to maintain cell uniformity throughout. To verify the one-dimensional behaviour of the proposed structure, on which the transmission-line theory was based, a single row of six cells was implemented and characterized using a network analyzer. The frequency response $\left(S_{21}\right)$, shown in Fig. 14, reveals the high-pass characteristic of the LH topology, with a cutoff (Bragg) frequency near $800 \mathrm{MHz}$. A loss of about $2.5 \mathrm{~dB}$, consisting of both mismatch and conductor losses, is apparent in the passband. Also note in Fig. 14, the cutoff region below $800 \mathrm{MHz}$ and the stopband, which starts forming beyond 2 GHz.

The 2-D LHM consisted of $11 \times 6$ unit cells and was etched alongside a $55 \mathrm{~mm} \times 50 \mathrm{~mm}$ parallel-plate waveguide, serving as the RHM. The parallel-plate waveguide was excited with a shorted vertical probe connected to an SMA connector. Matching chip resistors were used to terminate the cells at the LHM and parallelplate waveguide boundaries. The final

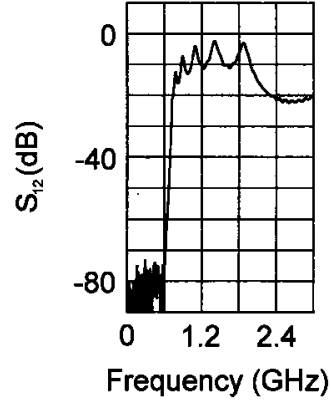

Fig. 14: Experimentally obtained frequency response of a 1-D LH structure; 6 cells of size $5 \mathrm{~mm}$ each.

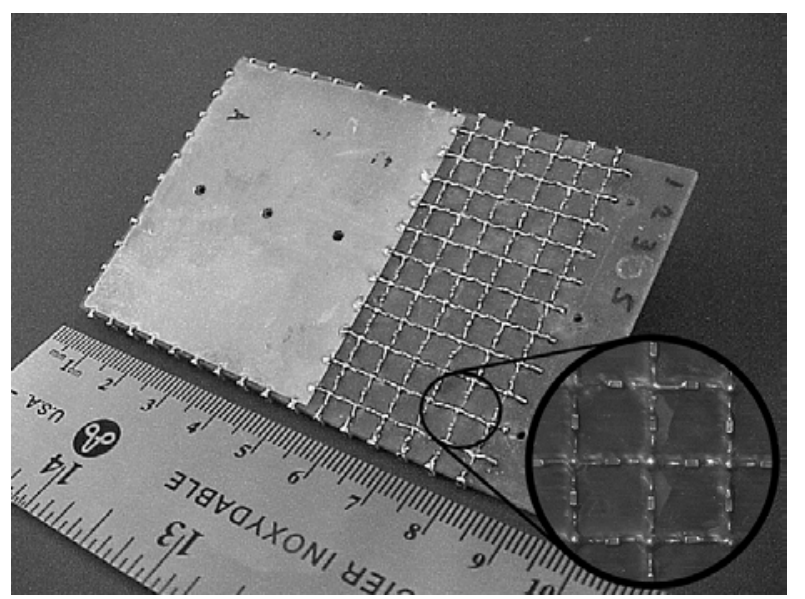

Fig. 15: Focusing device: LHM prototype interfaced with a parallel-plate waveguide $(60 \mathrm{~mm} \times 95 \mathrm{~mm})$. The inset magnifies the surface of the LHM unit cell.

prototype measures approximately $60 \mathrm{~mm} \times 95 \mathrm{~mm}$ and is shown in Fig. 15; the inset magnifies the unit cells.

\section{B. Experimental Results}

A near-field probing apparatus was employed to measure the vertical electric fields over the surface of the structure through proximity coupling. The excitation SMA connector was placed in the parallel-plate waveguide at the position of the central hole depicted in Fig. 15 . The detecting probe, essentially a short vertical dipole, was scanned across the $11 \times 6$-cell LHM by the probing apparatus in increments of $5 \mathrm{~mm}$ (calibrated at the inductor sites, approximately $1 \mathrm{~mm}$ above the device plane) by means of a stepper motor. Transmission scattering parameter readings were taken from $0-3 \mathrm{GHz}$ at each cell site using an HP8753D network analyzer.

The dispersion characteristic of the LHM was obtained from the experimental data using the average phase shift incurred per unit cell $(\beta d)$, and is presented in Fig. 16. This dispersion relation reveals a lower cut-off frequency (Bragg frequency) near $750 \mathrm{MHz}$ (modified from that of Fig. 14 due to the transition from one to two dimensions) and a region of propagation extending from approximately 1 to $2 \mathrm{GHz}$ that supports a backward wave. The region from 2 to $3 \mathrm{GHz}$ corresponds to a stopband, but is not depicted in Fig. 16 because the data is obscured due to strong mismatch losses at these frequencies. A comparison with the LHM dispersion relation 


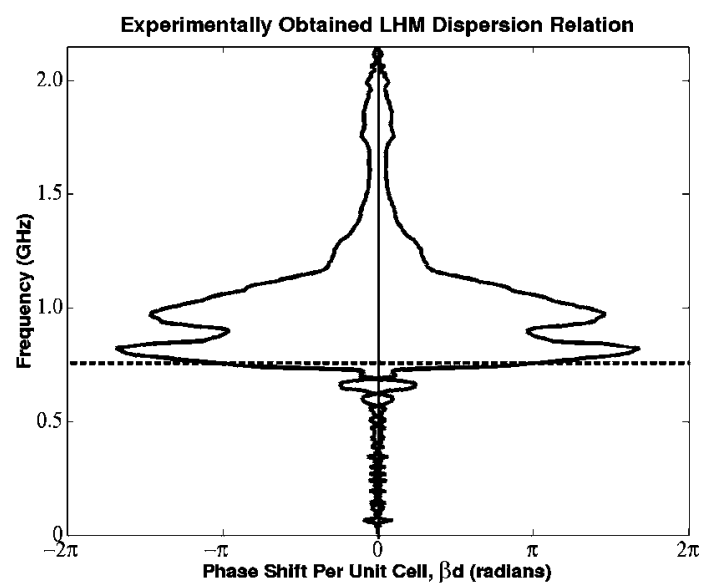

Fig. 16: Experimentally obtained LHM dispersion relation for the 2-D focusing device showing a cut-off frequency near $750 \mathrm{MHz}$ and a left-handed passband from approximately $1-2 \mathrm{GHz}$.

of Fig. 5 shows a qualitative correspondence, and suggests that this passband indeed supports a propagating backward spatial harmonic. Furthermore, this dispersion relation proportionally represents the index of refraction of the LHM structure through the propagation constant $\beta$. In the most well-defined region (approximately $1.3-1.9 \mathrm{GHz}$ ), the absolute refractive indexes range from approximately -5.5 to -1.2 . Since the parallel-plate waveguide possesses an absolute refractive index of 1.59 , the corresponding relative refractive indices vary from approximately -3.5 to -0.8 . It is also clear from the results of Fig. 15 that the proposed LHM structure exhibits an inherently wideband left handedness, conservatively placed at $60 \%$.

Fig. 17 shows a sample of the measured vertical $E$-field distributions over the $11 \times 6$-cell LHM at $1.55,1.65$, and 2.55 GHz. From Fig. 16, it is clear that the first two frequencies lie in the passband, whereas the last frequency occurs beyond this passband, inside the stopband. In accordance with theory, the field distributions at 1.55 and $1.65 \mathrm{GHz}$ indeed exhibit focusing, manifested by a localized region of increased transmission through the structure. Furthermore, the concavities of the wavefronts of Fig. 17 also indicate the convergent progression of phase associated with focusing. As expected, neither phenomenon is evident at $2.55 \mathrm{GHz}$.

The maximum focal amplitude obtained in the experiment was observed at a frequency of $1.65 \mathrm{GHz}$ (the central plot of Fig. 17), where the distinction between the peak and the two edges of the spot was noted to be approximately $15 \mathrm{~dB}$. However, it should be noted that the transmission amplitudes, when taken absolutely, are arbitrary since there are mismatch losses in coupling the input signal to the parallel-plate waveguide. Moreover, the method of proximity coupling measures fields that decay with distance in air above the device plane. Instead, the relative amplitudes of the peak and each of the two edges of the spot are more significant as a measure of merit, since they are indicative of the spot width. Fig. 18 makes a relative comparison of the full-wave-field simulation results and experimental results at $1.5 \mathrm{GHz}$, with the fields normalized to their maximum respective focal amplitudes in the LHM. The contours at -5 and

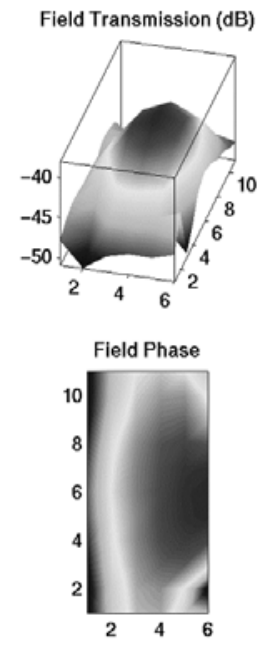

(a)
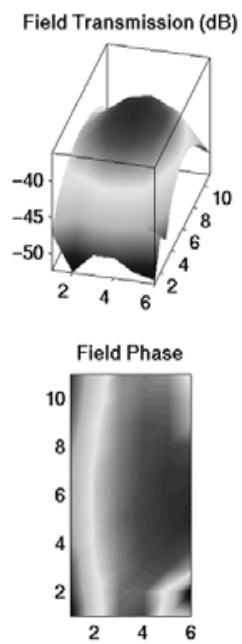

(b)
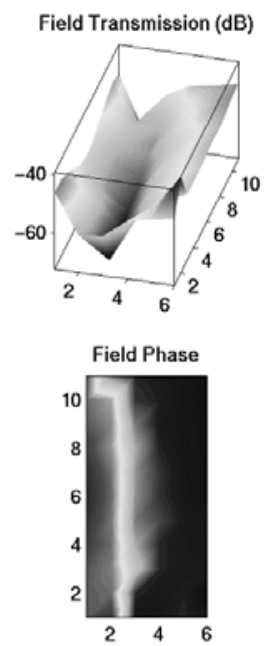

(c)
Fig. 17: Experimental data showing focal regions observed in the $11 \times 6$-cell LHM at: (a) 1.55 and (b) $1.65 \mathrm{GHz}$ (both within the LH passband of Fig. 16) and (c) the lack thereof at $2.55 \mathrm{GHz}$ (inside a stopband).

Normalized Field Transmission (dB) at $1.5 \mathrm{GHz}$ with Respect to Maximum Focal Amplitude

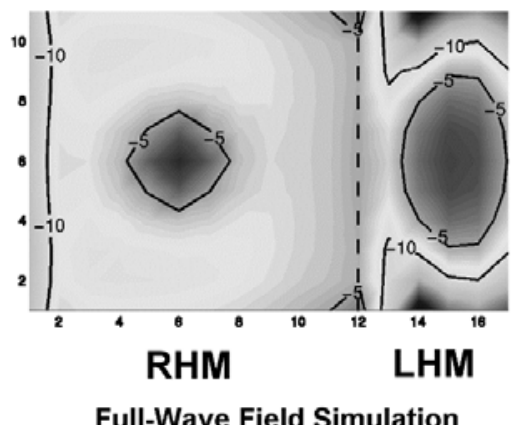

(a)

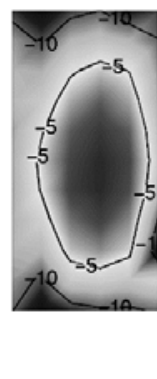

Experiment

(b)
Fig. 18: Correspondence of: (a) full-wave field simulation results and (b) experimental results at $1.5 \mathrm{GHz}$ (normalized to the maximum respective focal amplitudes in each case).

$-10 \mathrm{~dB}$ show agreement between the simulated and measured focal regions.

It is very interesting to point out that focusing of the RF power in the LHM is observed despite the electrically small extent of the LHM medium (one-sixth of a free-space wavelength at $1.65 \mathrm{GHz}$ ). This fact is suggestive of near-field focusing.

\section{CONCLUSION}

A technique has been presented for implementing planar media with an effective negative refractive index. The underlying concept is based on appropriately loading a printed network of transmission lines periodically with inductors and capacitors. This technique results in effective permittivity and permeability material parameters that are both inherently and simultaneously negative, obviating the need to synthesize these parameters separately, or by separate means.

The proposed media possess several other desirable features including very wide bandwidth over which the refractive index 
remains negative, the ability to guide 2-D TM waves, scalability from RF to millimetre-wave frequencies, low transmission losses, as well as the potential for tunability by inserting varactors and/or switches in the unit cell. The proposed concept has been verified with circuit and full-wave simulations. Moreover, a prototype focusing device has been implemented and tested experimentally. The experimental results demonstrated focusing of an incident cylindrical wave within an octave bandwidth and over an electrically short area; a phenomenon suggestive of near-field focusing.

We expect that new enabling $\mathrm{RF} /$ microwave devices can be implemented based on these proposed planar negative refractive index media for applications in wireless communications, surveillance, and radars.

\section{ACKNOWLEDGMENT}

The authors would like to thank Prof. K. G. Balmain, University of Toronto, Toronto, ON, Canada, for his encouragement and stimulating discussions.

\section{REFERENCES}

[1] V. G. Veselago, "The electrodynamics of substances with simultaneously negative values of $\varepsilon$ and $\mu$," Sov. Phys. Usp, vol. 10, no. 4, pp. 509-514, Jan.-Feb.1968.

[2] J. B. Pendry, A. J. Holden, D. J. Robins, W. J. Stewart, "Magnetism from conductors and enhanced nonlinear phenomena," IEEE Trans. on Microwave Theory and Tech., vol. 47, no. 11, pp. 2075-2084, Nov. 1999.

[3] D. R. Smith, W. J. Padilla, D. C. Vier, S. C. Nemat-Nasser, S. Schultz, "Composite medium with simultaneously negative permeability and permittivity," Phys. Rev. Lett., vol. 84, no. 18, pp. 4184-4187, May 2000 .

[4] R. A. Shelby, D. R. Smith, S. Schultz, "Experimental verification of a negative index of refraction," Science, vol. 292, 6 April 2001, pp. 77-79.

[5] R. E. Collin, Field Theory of Guided Waves, 2nd Ed., Toronto: WileyIEEE Press, 1990, pp. 16.

[6] S. Ramo, J. R. Whinnery, T. Van Duzer, Fields and Waves in Communication Electronics, 3rd Ed., Toronto: John Wiley \& Sons, 1994, pp. 263-264, 485, 250.

[7] J. B. Pendry, "Negative refraction makes a perfect lens," Phys. Rev. Lett., vol. 85, no. 18, pp. 3966-3969, Oct. 2000.

[8] R. W. Ziolkowski, E. Heyman, "Wave propagation in media having negative permittivity and permeability," Phys. Rev. E, vol. 64, pp. 056625/1-15, Nov. 2001.

[9] R. E. Collin, Foundations for Microwave Engineering, 2nd Ed., Toronto: McGraw-Hill, Inc., 1992, pp. 552-556, 570.

[10] D. M. Pozar, Microwave Engineering, 2nd Ed., Toronto: John Wiley \& Sons, 1998, pp. 424-427, 162.

[11] A. K. Iyer, G. V. Eleftheriades, "Negative refractive index metamaterials supporting 2-D waves," IEEE MTT-S International Microwave Symposium Digest (Seattle, WA), vol. 2, pp. 1067-1070, June 2-7, 2002.

[12] M. A. Tilston, K. G. Balmain, "A multiradius, reciprocal implementation of the thin-wire moment method," IEEE Trans. on Antennas and Propagat., vol. 38, no. 10, pp. 1636-1644, Oct. 1990.
George V. Eleftheriades (S'86-M'88-SM'02) received the Diploma (with distinction) in Electrical Engineering from the National Technical University of Athens, Greece in 1988, and the Ph.D. and M.S.E.E. degrees in Electrical Engineering from The University of Michigan, Ann Arbor, in 1993 and 1989 respectively.

From 1994 to 1997, he was with the Swiss Federal Institute of Technology in Lausanne, Switzerland, where he was engaged in the design of millimeter and sub-millimeter-wave receivers and in the creation of fast computer-aided design (CAD) tools for planar packaged microwave circuits. He is currently an Associate Professor with the Department of Electrical and Computer Engineering, University of Toronto, Toronto, ON, Canada. He has authored or co-authored over 70 papers in refereed journals and conference proceedings. His current research interests include negative refractive index metamaterials, integrated circuit (IC) antennas and components for broadband wireless communications, novel beam-steering techniques, low-loss silicon micromachined components, millimeter-wave radiometric receivers, and electromagnetic design for high-speed digital circuits.

Dr. Eleftheriades was a co-recipient of the 1990 Best Paper Award presented at the 6th International Symposium on Antennas (JINA) and the Ontario Premier's 2001 Research Excellence Award. His graduate students were the recipients of Student Paper Awards presented at the 2000 Antenna Technology and Applied Electromagnetics Symposium, the 2002 IEEE Microwave Theory and Techniques Society (IEEE MTT-S) International Microwave Symposium, and the 2002 IEEE International Symposium on Antennas and Propagation.

Ashwin K. Iyer ( $\mathrm{S}^{\prime} 01$ ) received the B.ASc. degree in electrical engineering from the University of Toronto, Toronto, ON, Canada, in 2001, and is currently working toward the Masters degree in electromagnetics at the University of Toronto.

In 1999 and 2000, he held summer positions as an Application-Specific Integrated Circuit (ASIC) designer for the Microlectronics Division and HighPerformance Optical Components Division, Nortel Networks, respectively. While with the University of Toronto, he has been involved with the development and characterization of RF/microwave metamaterials that exhibit a negative refractive index, also known as LHM.

Mr. Iyer was the second-place recipient of the Student Paper Competition of the 2002 IEEE Microwave Theory and Techniques Society (IEEE MTT-S) International Microwave Symposium (IMS), Seattle, WA. In 1998, he was the recipient of the Nortel Institute for Telecommunications Undergraduate Scholarship. In 2001, he was the recipient of the University of Toronto Centennial Thesis Award (electrical engineering) for his undergraduate work on balanced microwave amplifiers.

Peter C. Kremer was born in Neuenbuerg, Germany, on September 1937. Following a career with the Merchant Marine as Radio Officer he joined the Radio Astronomy Group of the University of Toronto, Toronto, ON, Canada, as Engineering Technologist in 1965. He worked on the development of low noise receivers, including Ruby and Rutile traveling-wave masers, digital and analog communication equipment for a long baseline interferometer, antenna measurement and design projects, and dual-band extremely-high-frequency (EHF) controlled-beam antenna for frequency-hopped signals. 